\title{
OTIOTOMTCS
}

Revista de economía, empresa y sociedad

PENSAMIENTO CRÍTICO

\section{Dirección de personas basada en evidencias. ¿Qué es, por qué importa, cómo implementarla?}

\author{
Fva Rimbau-Gilabert \\ Profesora de los Estudios de Economía y Empresa (UOC)
}

RESUMEN La dirección de personas basada en evidencias es una aproximación a la profesión y la práctica de la dirección de personas que se centra en aumentar el uso del pensamiento crítico y el uso de las mejores evidencias disponibles para tomar decisiones. Este artículo sugiere varias razones por las que los profesionales de la dirección de personas no adoptan un enfoque más basado en las evidencias y señala la utilidad que este enfoque puede tener para las organizaciones. Se presenta un proceso en siete etapas para tomar decisiones que hagan un mejor uso de las evidencias: identificar, preguntar, obtener, valorar, agregar, aplicar y evaluar. Existen cuatro fuentes de evidencias que pueden mejorar la calidad de las decisiones en dirección de personas: evidencia científica, datos de la organización, experiencia profesional y aportaciones de los stakeholders. Finalmente, se ofrecen sugerencias prácticas para la implementación de este enfoque en las organizaciones y se comentan las principales objeciones al mismo que suelen presentar los directivos.

PALABRAS CLAVE gestión basada en evidencias; recursos humanos; dirección de personas; pensamiento crítico; brecha investigación-práctica 


\title{
Evidence-based people management: what is it, why does it matter and how can it be implemented?
}

\begin{abstract}
Evidence-based people management is an approach to the profession and practice of people management that focuses on increasing the use of critical thinking and the best evidence available for decisionmaking. This article puts forward several reasons why people management practitioners do not adopt a more evidence-based approach and argues how useful this approach can be for organizations. A seven-stage process is presented for making decisions that make better use of evidence: identifying, asking, acquiring, appraising, aggregating, applying and assessing. There are four sources of evidence that can improve the quality of decisions in people management: scientific evidence, organizational data, professional experience and stakeholder input. Finally, practical suggestions are offered for implementing this approach in organizations, as well as the main objections presented by managers are outlined.
\end{abstract}

KEYWORDS Evidence-based management; human resources; people management; critical thinking; research-practice gap

\section{Introducción}

Por favor, indique para cada una de las siguientes afirmaciones si considera que es «cierta» o «falsa»:

1. La selección de nuevos empleados que tengan éxito en la organización es óptima cuando combina la valoración de los directivos con los resultados de tests validados.

2. Las personas incompetentes se benefician más del feedback que las personas muy competentes.

3. Ser inteligente es una desventaja para realizar trabajos poco cualificados.

4. Los tests de integridad no sirven porque la gente miente al responderlos.

¿Le sorprende saber que todas estas afirmaciones son falsas? Cada una de ellas ha sido refutada por una gran cantidad de estudios, pero eso no ha sido suficiente para que esta información llegue a la mayor parte de los directivos. En la práctica, muchos directivos -sin saberlo y con la mejor intención- actúan al gestionar 
personas en contra de las evidencias científicas y de los intereses de su propia empresa.

Hace tiempo que sabemos que esto ocurre. Un estudio realizado en Estados Unidos en 2002 identificó 35 hallazgos bien documentados en la investigación sobre recursos humanos y decidió comprobar el conocimiento de esa función entre 959 directivos (Rynes, Colbert y Brown, 2002). Estos directivos tenían que indicar si las afirmaciones propuestas sobre los hallazgos de la investigación eran correctas o no. Los resultados fueron chocantes: en promedio, los participantes solamente eligieron la respuesta correcta en un $57 \%$ de las ocasiones. Y en una réplica realizada en los Países Bajos (Sanders, Van Riemsdijk y Groen, 2008) la cosa no fue mucho mejor: solamente un 62 \% de las respuestas fueron correctas. Reconozcámoslo: estos resultados son solo algo mejores que los que obtendríamos al decidir a cara o cruz. La comunidad de la dirección de personas parece estar, en general, pobremente informada sobre qué nos dicen las evidencias científicas en áreas fundamentales como la selección, la formación, el feedback y las estrategias de recursos humanos.

De estas investigaciones surgió otro dato relevante: los directivos que obtenían mejores resultados eran los que tenían un mayor nivel educativo, leían más sobre dirección de personas y tenían una actitud más positiva hacia los hallazgos de la investigación académica. Es decir, que no se basaban solamente en su instinto o su experiencia para tomar decisiones, sino que añadían otra información que les ayudaba a decidir de forma menos sesgada. Estos directivos eran los avanzados de una tendencia en gestión de empresas de la que se habla en otros países desde hace unos años y que por fin está llegando a nuestro país: la gestión basada en evidencias.

En este artículo se presentan las ideas básicas de la gestión basada en evidencias y cómo se aplica al contexto de la dirección de personas. Se explica qué es, por qué preocuparnos por ella, a qué nos referimos por «evidencias» y cómo implantarla en nuestra organización. Por cierto, si le interesa conocer la respuesta al test con que comienza el artículo, puede encontrarlas en el anexo final.

\section{Qué es la dirección de personas basada en evidencias y por qué importa}

La expresión «basado en evidencias» se acuñó en la década de los años setenta en el campo de la medicina (aunque su uso no se extendió hasta los noventa), pero sus principios se aplican hoy en disciplinas tan variadas como la educación, la criminología, las políticas públicas, el trabajo social y -recientemente- la gestión. En cualquiera de estas áreas, la práctica basada en evidencias se centra en lo mismo: 
tomar mejores decisiones utilizando el pensamiento crítico y las mejores evidencias empíricas disponibles. La gestión basada en evidencias es una aproximación a la toma de decisiones y al trabajo diario de los directivos que les ayuda a evaluar en qué grado pueden confiar en las evidencias que tienen a mano, y que les ayuda a identificar, encontrar y evaluar evidencias adicionales relevantes para sus decisiones (Barends, Rousseau y Briner, 2014, pág. 4). En el campo de la dirección de personas, este enfoque se concreta en los «recursos humanos basados en evidencias» (evidence-based human resources, EBHR) o "dirección de personas basada en evidencias», que se centra en utilizar el pensamiento crítico y las mejores evidencias disponibles para tomar decisiones relacionadas con la profesión y la práctica de la dirección de personas.

Aunque todos los directivos utilizan evidencias en sus decisiones, muchos apenas prestan atención a la calidad de dichas evidencias (Barends, Rousseau y Briner, 2014). Es decir, a menudo toman malas decisiones que se basan en creencias con poco o ningún fundamento, modas e ideas popularizadas por gurús de la gestión. En el campo de la dirección de personas, esto se traduce en una amplia variedad de situaciones poco deseables. Por ejemplo, no seleccionar a los mejores candidatos, dificultar que los empleados mejoren su rendimiento por no proporcionarles el feedback necesario, no detectar potenciales problemas de integridad personal, tener elevadas tasas de rotación de personal o de ausentismo, etc. Además, se dedican grandes importes a programas novedosos que, en el mejor de los casos, no producirán un retorno o que, en el peor caso, pueden llegar a ser dañinos para las organizaciones, sus empleados y sus clientes. A todo esto se añade el problema de que resultará imposible explicar por qué las cosas no han salido cómo se esperaba, con lo que la mejora será improbable.

Las razones por las que esto ocurre son múltiples, y tanto los profesionales como los académicos deben reconocer su parte de responsabilidad. En primer lugar, como Jeffrey Pfeffer (citado en Jacobs, 2015) señala, a menudo los departamentos de recursos humanos se centran más en sus «programas» que en pensar de forma crítica. Muchas empresas ponen programas en marcha porque otras empresas lo están haciendo o porque una consultoría se lo sugiere, pero no preguntan antes «¿seguro que tenemos un problema?» y, en caso afirmativo, «¿qué evidencia tenemos de que este programa puede ser una solución?».

En segundo lugar, el conocimiento científico no llega a los profesionales. Para empezar, los académicos suelen escribir en revistas destinadas a otros académicos, en un lenguaje poco accesible para los profesionales. Además, en algunas áreas no existe consenso académico sobre qué es lo que «funciona»-ya que se describen correlaciones, pero no causalidades-, lo que hace desconfiar a los profesionales. Son necesarias más publicaciones divulgadoras de buena calidad, que estén a medio camino entre la academia y la práctica -como Oikonomics-, que 
realmente den apoyo a los profesionales y les expliquen lo que necesitan saber sobre las conclusiones de la investigación en términos comprensibles.

Finalmente, también es necesario reconocer que los profesionales no reciben formación sobre cómo desarrollar una práctica basada en evidencias. En el siguiente apartado explicamos un proceso para tomar mejores decisiones con base en las evidencias disponibles.

\section{El proceso de la gestión basada en las evidencias}

El Center for Evidence-Based Management (CEBMa, 2014, pág. 4) sugiere seguir un proceso en seis etapas (las seis «A» en inglés) para tomar mejores decisiones, al que añadimos una etapa inicial de identificación:

1. Identificar: detectar un problema que necesita solución o una oportunidad que puede reportar beneficios.

2. Preguntar («Asking»): traducir esa cuestión práctica o problema en una pregunta que sea posible responder.

3. Obtener («Acquiring»): buscar y recopilar las evidencias sistemáticamente.

4. Valorar («Appraising»): valorar críticamente el nivel de confianza que merecen las evidencias y su relevancia.

5. Agregar («Aggregating»): ponderar y combinar las evidencias.

6. Aplicar («Applying»): incorporar las evidencias al proceso de toma de decisiones.

7. Evaluar («Assessing»): evaluar el resultado de la decisión tomada.

\subsection{Identificar}

Una de las críticas más frecuentes a la dirección de personas no basada en evidencias es que se centra en implementar soluciones para problemas que no han sido verificados, que quizá ni siquiera existen. Es el llamado solution-eering: una solución (quizá promovida por consultorías externas) en busca de un lugar donde implantarse, incluso aunque no haya ningún problema que solucionar.

Una práctica basada en evidencias debe verificar que existe un problema que genera ineficiencias o una oportunidad que puede aportar beneficios. Por ejemplo, la sensación de que el ausentismo es elevado no es suficiente para implementar un nuevo programa que intente mejorarlo. Antes de iniciar cualquier acción, deberíamos recopilar evidencias que muestren: a) que los niveles de ausentismo son altos o han aumentado, b) que hay posibilidad de mejora en los niveles de ausentismo 
sin incurrir en costes excesivos, por medio de identificar las áreas de la empresa en que esta mejora es más factible, y c) que existen intervenciones que podemos implementar y que han mostrado su eficacia para reducir el ausentismo. Hablaremos de cómo podemos obtener estas evidencias más adelante.

\subsection{Preguntar}

Una vez identificado el problema que debemos resolver, es necesario comenzar con una pregunta concreta basada en un problema práctico. Una pregunta como «¿Funcionan los programas de incentivos?» resulta interesante pero es demasiado amplia. Es necesario tener en cuenta en qué tipo de organización estamos y los tipos de resultados que interesa obtener para concretar la pregunta. Así, la pregunta anterior podría reformularse en «Los programas de incentivos ¿mejoran la motivación de los trabajadores?» o «¿mejoran la productividad?» o «¿reducen el absentismo?», según cuál sea el problema que la empresa necesita resolver. Una pregunta bien formulada es un problema medio resuelto, dado que canaliza los esfuerzos en la dirección que con mayor probabilidad puede ofrecer resultados significativos.

\subsection{Obtener}

Un elemento fundamental de la dirección de personas basada en evidencias es, precisamente, obtener evidencias que nos ayuden a responder la pregunta formulada. Habitualmente, los directivos recurren a su propia experiencia profesional o a la de otros directivos, o bien al consejo de consultores externos. No obstante, estas evidencias no son las únicas disponibles, hay que contar también con la evidencia científica, los datos provenientes de la propia organización, y las opiniones de los stakeholders.

Supongamos que hemos detectado un problema de ausentismo en nuestra empresa. La pregunta que nos planteamos es ¿cómo se puede reducir el ausentismo en esta empresa? Veamos qué puede aportar cada fuente de evidencias.

\section{Evidencia científica}

Los resultados de investigaciones científicas empíricas publicados en revistas académicas pueden indicar cuáles son las principales causas del ausentismo y qué intervenciones pueden ser eficaces. También pueden proporcionar las ratios de ausentismo registradas para mi sector y mi localización, lo que me ayuda a determinar si mi tasa actual es realmente elevada o no. 
Para acceder a la literatura científica, se puede contactar con expertos en el tema (a menudo, profesores universitarios) que puedan indicar directamente las referencias bibliográficas más relevantes. Si esto no es posible, se puede buscar en alguna de las múltiples bases de datos que recopilan el texto completo de revistas científicas, a las que se puede tener acceso mediante acuerdos con las bibliotecas universitarias o a través de las propias compañías propietarias de las bases de datos. Por ejemplo, ABI/Inform es una buena referencia donde buscar activando la opción «Evaluado por expertos». Una opción alternativa, gratuita pero que filtra menos la información y ofrece peor acceso a los artículos, es Google Scholar.

\section{Evidencia organizativa}

Llevar un registro en la propia organización permitirá saber cuál es realmente la tasa de ausentismo, qué tipo de ausencias (largas o cortas) tenemos y dónde se producen, podremos compararlas con la media del sector y ver si se dibuja una tendencia. También facilitará elegir en qué áreas de la empresa interesa más intervenir y permitirá calcular los posibles costes y beneficios de una intervención.

No se trata de acumular grandes cantidades de informes y registros internos, ni de adquirir un sofisticado sistema de people analytics, sino de detectar un problema y ver qué datos serán necesarios para tomar una decisión bien fundamentada. Hay áreas en la función de recursos humanos que se prestan especialmente bien a su gestión basada en evidencias, como las de selección y reclutamiento.

\section{Evidencia experiencial}

La experiencia y el criterio de los profesionales es importante, puesto que con los años acumulan un conocimiento tácito sobre lo que funciona en su organización, que es valioso incorporar en la toma de decisiones. Esta experiencia puede responder a preguntas sobre qué ha funcionado en el pasado y por qué.

A pesar del valor otorgado a la experiencia, el hecho de que una intervención haya funcionado en una organización no garantiza que funcione en otra. Esto significa que no es recomendable basarse en el benchmarking y las llamadas «mejores prácticas» para tomar decisiones. Barends y otros (2014, pág. 6) destacan que «los profesionales a veces copian lo que hacen otras organizaciones sin evaluar críticamente si dichas prácticas son realmente eficaces y, en caso de serlo, si es probable que también funcionen en un contexto diferente. El benchmarking puede mostrar formas alternativas de hacer las cosas, pero no es necesariamente un buen indicador de lo que funcionaría en otro lugar». La necesidad de evaluar críticamente las evidencias se pone aquí de manifiesto con mayor intensidad, si cabe, que con otras fuentes de evidencias. 


\section{Evidencia de los stakeholders}

En cualquier decisión, es necesario incorporar los valores y preocupaciones de las personas y organizaciones que se verán afectadas por la misma. Ante el problema de ausentismo, habría que averiguar qué piensan los empleados y los directivos sobre las intervenciones propuestas para reducirlo, si creen que pueden tener inconvenientes o consecuencias negativas inesperadas. Esta fuente de evidencias incorpora un criterio ético imprescindible que genera intervenciones aceptables y consideradas legítimas entre los afectados, pero también un criterio práctico, que destaca qué es factible teniendo en cuenta a los que van a implementar o recibir los efectos de la decisión.

\subsection{Valorar las evidencias críticamente}

Las evidencias de cualquiera de las anteriores fuentes nunca son perfectas. En todos los casos, es necesario evaluar cómo de fiable y de relevante es la información obtenida y escrutar posibles sesgos. Por ejemplo: un estudio puede ofrecer conclusiones atractivas pero estar basado en una muestra pequeña de un sector muy diferente al nuestro; la opinión de un colega sobre la eficacia de una intervención puede estar basada en una anécdota; la forma de hacer las cosas hasta el momento en nuestra organización puede no haber sido evaluada nunca para ver si funciona realmente o no.

La clave es valorar críticamente toda fuente de evidencias mediante preguntas como: ¿dónde y cómo se obtuvieron estas evidencias?, ¿son las mejores evidencias disponibles?, ¿tenemos suficientes evidencias como para alcanzar una conclusión?, ¿hay razones para pensar que estas evidencias pueden estar sesgadas en una dirección en particular? En el caso de las publicaciones científicas, existe una clara escala que jerarquiza los distintos tipos de estudios según su fiabilidad. Puede aprenderse más sobre ello en CIPD (2016).

Para algunas decisiones, puede ser que no exista literatura científica sobre el tema y que no haya datos organizativos de ningún tipo. Entonces no habrá más opción que basar la decisión en la experiencia profesional del equipo decisor o hacer una prueba piloto de diferentes opciones y ver qué funciona mejor. Para aprender a realizar experimentos en la propia organización, véase Gray (2004) y Davenport (2009). En cualquier caso, utilizar estas evidencias de calidad limitada puede llevar a mejores decisiones que utilizar solamente la intuición, siempre y cuando al utilizarlas seamos conscientes de sus limitaciones. 


\subsection{Agregar}

Cuando se han recopilado las mejores evidencias posibles, es necesario analizarlas en su conjunto y deducir en qué sentido nos orienta cada una, sin olvidar de hacer explícitas las posibles contradicciones entre ellas y los límites a su fiabilidad o su aplicabilidad a nuestro caso concreto. Una visión integrada ponderará las evidencias obtenidas de los estudios científicos, la propia experiencia y el contexto para llegar a conclusiones sobre la pregunta planteada y recomendar la actuación más adecuada ante el problema concreto de la organización.

\subsection{Aplicar a la decisión}

Tener evidencias no es lo mismo que tener respuestas. Los directivos aún necesitan interpretar las evidencias disponibles a la luz de la situación en que se encuentran. Como destacan Rousseau y Gunia (2016), esto puede ser particularmente complejo cuando las evidencias existentes no provienen del mismo sector, campo o disciplina, o no responden exactamente a las preguntas planteadas. Por ejemplo, es posible tener evidencias obtenidas en el sector hospitalario pero que nuestra empresa sea hotelera; o bien que haya conclusiones relativas a la calidad del producto cuando nuestra pregunta se refería al coste. También puede ser que la evidencia científica sobre intervenciones posibles no sea lo suficientemente detallada como para guiar su implantación práctica, o que no sea posible trasladar directamente los experimentos realizados en un contexto controlado como el de la investigación científica al contexto real de una organización. En situaciones así, aplicar esas evidencias a la decisión que nos ocupa puede ser complejo, y requerirá un esfuerzo de adaptación relevante.

\subsection{Valorar los resultados}

Evaluar los resultados de las decisiones tomadas es inherente a una práctica basada en las evidencias. Es necesario recopilar información sobre el impacto de las intervenciones realizadas, conversar y reflexionar sobre ellas para alcanzar conclusiones sobre cómo proceder en el futuro, tanto respecto al problema que suscitó la decisión como sobre el propio proceso de utilizar las evidencias y mejorar la práctica. 


\section{Consejos para llevarlo a la práctica}

Cambiar el modo en que se toman decisiones en una organización no es algo sencillo. Los profesionales de cualquier nivel pueden sentirse incómodos o incluso amenazados cuando se cuestionan los supuestos que siempre se han dado por sentados, o cuando se les piden evidencias que apoyen sus propuestas. A continuación comentaremos algunas sugerencias prácticas para ir introduciendo el enfoque basado en evidencias en la propia organización, así como respuestas a las principales objeciones de los directivos a este enfoque.

David D'Souza (2017) propone los siguientes consejos para la persona que intenta implantar el uso de evidencias para la toma de decisiones en su organización:

1. Hacer sentir a la gente que se está perdiendo algo: hay que destacar organizaciones que utilizan un enfoque más basado en evidencias y mostrar los excelentes resultados y el reconocimiento que están obteniendo. Así se genera la sensación de que si no se hace lo mismo nos estaremos quedando atrás. Google es un ejemplo bien conocido en este sentido.

2. Destacar la posibilidad de elegir: a nadie, y en particular a los directivos, le gusta pensar que no tiene elección. Por eso es recomendable ofrecer varias opciones razonables y pedir que se elija una. Lo importante no es llevarse el mérito de una decisión, sino lograr que las acciones que se emprendan sean lo más acertadas posible.

3. Realizar una prueba de bajo coste: para facilitar que se acepte una propuesta, es mejor minimizar las inversiones necesarias en tiempo y dinero. Por ejemplo, pedir «solo una hora» para experimentar con una nueva forma de evaluar críticamente una decisión y revisar las evidencias disponibles.

4. Centrarse en un fracaso o problema reconocido pero no sensible: lo más molesto que puede hacerse es apuntar al último gran fracaso de la organización, ya que todavía habrá egos dañados a los que no les gustará escuchar que «mi enfoque lo habría evitado». Es recomendable elegir temas lo suficientemente antiguos como para que haya una distancia emocional y sobre los que haya un consenso en que se adoptó un enfoque equivocado, o bien atacar problemas organizativos reconocidos (del tipo «falta tiempo, faltan recursos, falta eficiencia») y proponer una solución. Se trata de señalar cómo podría evitarse o mejorarse la situación en el futuro, más que destacar las razones del fracaso reciente.

5. Seleccionar los aliados con gran cuidado: es necesario ir ganando aliados o defensores del enfoque y crear una corriente de aprobación. Se puede hablar con otras personas antes de las reuniones para explicar de qué trata la propuesta y pedir apoyo, de modo que al proponer una forma de actuar ya haya un coro de voces que dicen "ya hemos hablado de ello y estamos de acuerdo". 
Por otra parte, es habitual que los directivos crean que un enfoque basado en evidencias presenta inconvenientes que lo hacen inaplicable:

1. Tiempo. Es frecuente que los directivos crean que la búsqueda y evaluación de evidencias les tomará un tiempo excesivo del que no disponen. No obstante, no todas las decisiones (de hecho muy pocas) de dirección se toman de un día para el otro, sino que siguen un proceso de maduración que permite la incorporación crítica de evidencias.

2. Datos. Los datos organizativos necesarios para una decisión pueden ser difíciles de conseguir, o bien los datos disponibles pueden ser de baja calidad, con lo que se reduce la capacidad de incorporar la información interna como fuente de evidencias. La respuesta a esta objeción es ir planteando una mejora progresiva en la calidad de los datos recabados, no rechazar de plano el enfoque basado en evidencias.

3. Capacidad de análisis cuantitativo. Relacionado con lo anterior, los profesionales de recursos humanos a menudo no han desarrollado la capacidad analítica necesaria para interpretar los datos y alcanzar conclusiones útiles para la organización. Dado que cada vez hay más organizaciones orientándose hacia una gestión basada en la evidencia, es recomendable que los profesionales desarrollen la capacidad de analizar datos y realizar inferencias a partir de ellos, lo que les puede favorecer en su trabajo actual o en el próximo.

\section{Conclusión}

La dirección de personas basada en evidencias no es un punto de llegada, sino un medio de transporte que nos lleva más directamente al destino que elijamos. Es un cambio de mentalidad en el modo cómo se decide sobre las políticas y prácticas de recursos humanos en una organización, que permite distinguir el grano de la paja entre los numerosísimos programas que podemos elegir. Facilita la implementación de los programas seleccionados y mejora los resultados que obtenemos de ellos.

Para lograrlo, es necesario que las organizaciones utilicen sistemáticamente el pensamiento crítico y se abran al aprendizaje. Además, requiere un acercamiento entre el mundo profesional y el académico, con esfuerzo por ambas partes. Los profesionales de la dirección de personas deben buscar la objetividad en sus decisiones y estar dispuestos a cambiar su forma de pensar y actuar cuando las evidencias sugieran que esta era inadecuada. Por su lado, los académicos deben ser capaces de trasladar sus hallazgos a la práctica profesional, para lo que necesitan comprender las condiciones en que esta se desarrolla. 


\section{Anexo: solución al test}

El cuestionario inicial se ha obtenido de Rousseau y Barends (2011). Aquí anotamos lo que la evidencia científica indica respecto a cada afirmación. Para saber más de cada uno de estos aspectos -y otros muchos-, recomendamos consultar Locke (2011).

1. «La selección de nuevos empleados que tengan éxito en la organización es óptima cuando se combinan la valoración de los directivos con los resultados de tests validados». Evidencia: añadir la valoración de los directivos a las decisiones de selección de hecho lleva a peores decisiones de selección que usar únicamente tests e indicadores de selección validados.

2. «Las personas incompetentes se benefician más del feedback que las personas muy competentes». Evidencia: las personas incompetentes tienen una gran dificultad para entender el feedback y tienden a utilizarlo de modo menos eficaz que sus compañeros/as más inteligentes.

3. "Ser inteligente es una desventaja para realizar trabajos poco cualificados». Evidencia: los trabajadores más inteligentes suelen tener un mejor desempeño en general, con independencia de si el trabajo es diseñar un hotel o limpiar sus habitaciones.

4. "Los tests de integridad no funcionan porque la gente miente al responderlos". Evidencia: aunque la gente ciertamente distorsiona sus respuestas, los tests de integridad siguen siendo muy buenos predictores de comportamientos laborales disfuncionales como el robo.

\section{Referencias bibliográfícas}

BARENDS, E.; ROUSSEAU, D. M.; BRINER, R. B. (2014). Evidence-Based Management. The Basic Principles. Amsterdam: Center for Evidence-Based Management [artículo en línea]. [Fecha de consulta: 1 de marzo de 2017]. <http:// www.cebma.org/wp-content/uploads/evidence-based-practice-the-basicprinciples.pdf>

CIPD (2016). «In search of the best available evidences». Positioning paper [artículo en línea]. Londres: Chartered Institute of Personnel and Development. [Fecha de consulta: 10 de junio de 2017]. <https://www.cipd.co.uk/Images/in-searchof-the-best-available-evidence_tcm18-16904.pdf>

DAVENPORT, T. H. (2009) «How to design smart business experiments». Harvard Business Review. Febrero, págs. 69-76 [artículo en línea]. [Fecha de consulta: 
10 de junio de 2017]. <https://hbr.org/2009/02/how-to-design-smart-business-experiments>

D'SOUZA, D. (2017, 27 de enero). "Sell the sizzle, not the evidence-based sausage». Future Work Centre [artículo en línea]. [Fecha de consulta: 1 de marzo de 2017]. <http://www.futureworkcentre.com/2017/01/sell-the-sizzle-not-theevidence-based-sausage/>

GRAY, D. E. (2004). «Experimental and quasi-experimental reseach design». En: Doing research in the real world (cap. 4, págs. 67-97). Londres: Sage Publications.

JACOBS, K. (2015). «Under the microscope». HR Magazine. Enero, págs. 24-31.

LOCKE, E. (ed.) (2011). Handbook of principles of organizational behavior: indispensable knowledge for evidence-based management. Nueva York: John Wiley \& Sons.

PFEFFER, J.; SUTTON, R. I. (2006). Hard facts, dangerous half-truths, and total nonsense: profiting from evidence-based management. Cambridge, MA: Harvard Business Press.

ROUSSEAU, D. M. (2006). «ls there such a thing as 'evidence-based management'?». Academy of Management Review. Vol. 31, n. ${ }^{\circ}$ 2, págs. 256-269.

ROUSSEAU, D. M.; BARENDS, E. G. (2011). «Becoming an evidence-based HR practitioner». Human Resource Management Journal. Vol. 21, n. ${ }^{\circ}$ 3, págs. 221 235.

ROUSSEAU, D. M.; GUNIA, B. C. (2016). «Evidence-based practice: the psychology of EBP implementation». Annual Review of Psychology. Núm. 67, págs. 667-692.

RYNES, S. L.; COLBERT, A. E.; BROWN, K. G. (2002). «HR professionals' beliefs about effective human resource practices: correspondence between research and practice». Human Resource Management. N. ${ }^{\circ} 41$, págs. 149-174.

SANDERS, K.; VAN RIEMSDIJK, M.; GROEN, B. (2008). "The gap between research and practice: a replication study on the HR professionals' beliefs about effective human resource practices». The International Journal of Human Resource Management. Vol. 19, n. 10, págs. 1976-1988. 


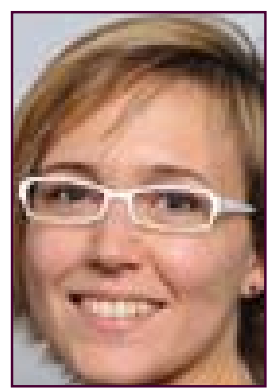

\section{Fva Rimbau-Gilabert erimbau@uoc.edu Profesora de los Estudios de Economía y Empresa (UOC)}

Doctora en Administración y Dirección de Empresas por la Universidad de Barcelona. Es profesora de la UOC desde el año 2004, donde enseña en los ámbitos de la dirección de personas, la gestión del cambio y la responsabilidad social corporativa. Desde 2014, además, es miembro de la Comisión Académica del Programa de Doctorado en Información y Sociedad del Conocimiento. Anteriormente, ejerció como directora académica de diversos programas: la Licenciatura en Ciencias del Trabajo, el Máster en Prevención de Riesgos Laborales y el Área de Posgrado de los Estudios de Economía y Empresa.

En cuanto a la investigación, sus líneas de interés son las siguientes: organización flexible del trabajo, específicamente el teletrabajo y los contratos contingentes; las relaciones entre la digitalización, la dirección de personas y el bienestar de los empleados; el comportamiento organizacional y el aprendizaje en línea, en particular, el aprendizaje en línea de competencias interpersonales, los equipos virtuales en la educación en línea, y el liderazgo y el bienestar de los empleados en las universidades en línea.

Eva es miembro del Digital Business Research Group (DigiBiz), que ha sido reconocido como Grupo de Investigación Consolidado de la Generalitat de Cataluña. Difunde su investigación en revistas indexadas, congresos internacionales y capítulos de libro de editoriales de prestigio. Ha participado en proyectos de investigación tanto de ámbito local como nacional e internacional, y actúa regularmente como revisora externa para revistas internacionales en sus áreas de especialización. Está acreditada como profesora lectora de la Agencia de Calidad Universitaria catalana (AQU).

Los textos publicados en esta revista están sujetas -salvo que se indique el contrario- a una licencia de Reconocimiento 3.0 España de Creative Commons. Podéis copiarlos, distribuirlos, comunicarlos públicamente y hacer obras derivadas siempre que reconozcáis los créditos de las obras (autoría, nombre de la revista, institución editora) de la manera especificada por los autores o por la revista. La licencia completa se puede consultar en http://creativecommons.org/licenses/by/3.0/es/deed.ca.

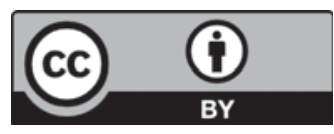

